\title{
How quarantine and social distancing policy can suppress the outbreak of novel coronavirus in developing or under poverty level countries : a mathematical and statistical analysis.
}

\author{
Atiqur Chowdhury ( $\nabla$ smatiqc@nmsu.edu ) \\ New Mexico State University https://orcid.org/0000-0002-9130-1911 \\ K M Ariful Kabir \\ Kyushu Daigaku \\ Jun Tanimoto \\ Kyushu Daigaku
}

\section{Research Article}

Keywords: Social Distancing Mathematical model, Basic reproduction number, COVID-19 pandemic, Next generation matrix, Transmissibility

Posted Date: May 14th, 2020

DOI: https://doi.org/10.21203/rs.3.rs-20294/v2

License: (c) (1) This work is licensed under a Creative Commons Attribution 4.0 International License.

Read Full License 


\title{
How quarantine and social distancing policy can suppress the outbreak of novel coronavirus in developing or under poverty level countries : a mathematical and statistical analysis
}

\author{
Atiqur Chowdhury ${ }^{1 * \dagger}, \mathrm{K}$ M Ariful $\mathrm{Kabir}^{2,3 \dagger}$ and Jun Tanimoto ${ }^{2,4 \dagger}$
}

\footnotetext{
${ }^{*}$ Correspondence:

smatiqc@nmsu.edu

${ }^{1}$ College of Business, Economics, Applied Statistics and

International Business, New Mexico

State University, MSC 3CQ, PO

Box 30001 Las Cruces,NM

88003-8001, U.S.A

Full list of author information is available at the end of the article

$\dagger$ Equal contributor
}

\begin{abstract}
Background: COVID-19 is a transmissible viral disease that has spread around the world rapidly and is currently a significant thread to developing and impoverished country by the World Bank and WHO's prediction. Without inventing vaccination or the proper treatment, how we control the transmission of the COVID-19 is one of the most important questions with which peoples are facing right now. By the WHO's guidelines, some policies termed as isolation, quarantine, lockdown, and social distancing would give a stunning direction to control the epidemic outbreak.
\end{abstract}

Methods: In this paper, we developed a mathematical model named "Social distancing SEIQR model" to reduce the basic reproduction number RO by combining both quarantine and social distancing parameters based on the real cases where medical equipment and other resources are limited.

Results: Our modeling basic reproduction number $R_{0}$ is an almost accurate predictor threshold to assess the transmissibility of the COVID-19 in Bangladesh. Our modeling basic reproduction number R0 is an almost accurate predictor threshold to assess the transmissibility of the COVID-19 in Bangladesh. Our study result showed, our model fitted well with the numerically simulated results to the reported COVID-19 cases data for Bangladesh by a linear regression polynomial fit analysis.

Conclusion: Our model will help to find strategies to reduce the human-to-human transmission of the virus and protect the nation when a country has limited medical resources.

Keywords: Social Distancing Mathematical model; Basic reproduction number; COVID-19 pandemic; Next generation matrix; Transmissibility

\section{Background}

COVID-19 is a transmissible viral disease that has stroke around the world rapidly and is currently a significant thread to developing and impoverished country by the World Bank and WHO's prediction [1], [2], [3], [4] .Other than developed countries like the U.S.A, China, Japan, Canada, Germany, and United Kingdom, the reproduction level of this coronavirus is faster in the developing countries like Bangladesh [5],[6]. Without inventing vaccination or proper treatment, how we control the transmission of the COVID-19 is one of the most admitted questions with that people are facing right now. Until such treatments are 
developed, some policies termed as isolation, quarantine, lockdown, and social distancing would give a stunning direction to control the epidemic outbreak.

Merging those ideas, theoretical epidemic models, and analyses that give some shed of light of infectious diseases to control the risk of infection and transmission gets public attention. Therefore, the SEIQR epidemic model deliberated to study the novel coronavirus by introducing the social distancing parameter as a case study of Bangladesh. To date, no study has conducted.

The compartment model, with a mean-field approximation, is known to be the most usual analytical method for recognizing the complicated disease dynamics model. The simplest disease model is denoted by the SI pattern [7], where ' $S$ ' represents susceptible individuals and ' $\mathrm{I}$ ' represents infectious individual. An extended concept built as a SIS epidemic model [8] to reflect an infected person, who once again becomes susceptible. Furthermore, a susceptible-infected-recovered (SIR) model used to analyze and predict the demanding amount of vaccination, medical assistance and facilities during the epidemic period by many researchers [8]. Correspondingly, the SEIR model [9][11] (susceptible-exposed-infected-recovered) and the SEIQR model (susceptible-exposedinfected-quarantine-recovered) model [12] are extensions of the SIR model for various diseases and situations that explained the pre-infection exposed state and the post quarantine policy. For example, Kissler et al. [23] projecting the transmission dynamics of SARS-CoV2 through the post-pandemic period, which presumed SEIRS (that indicates they presumed immunity might lose) considering two-strain (to confirm cross-immunity effect between the two strains). The model validated by the real data of HCo-Co42 and HCoV-HKU1.

Also, many researchers investigate epidemic models by introducing metapopulation, vaccination, information spreading, and treatment [13]-[18]. Recently, Chen et al. [19] explained Bats-Hosts-Reservoir based mathematical model for novel coronavirus that does not seem concerned about any provisions to suppress the pandemic. Additionally, the area of such various studies has been extended by many investigators to incorporate exposed and quarantine provisions to protecting minimize the risk of infection [20]-[26]. In this paper, we developed a mathematical model named "Social distancing SEIQR model" to reduce the basic reproduction number $R_{0}$ by combining both quarantine and social distancing parameters based on the real cases that observed where medical equipment and others resources are limited.

The paper organized as follows. First, we introduced a method and model in detail, followed by estimating parameters and analyzing the reproduction numbers. Then, we investigated the proposed model through numerical simulations and statistical simulations. Then, we discussed our findings, and in the end, provided concluding notes and restrictions of our findings that need further discussion.

\section{Method}

We introduce a modified infectious disease dynamics model (SEIR model) by introducing the quarantine $(\mathrm{Q})$ and social distancing for modeling and reducing the transmission of the novel coronavirus in Bangladesh.

To formulate the model into the social distancing SEIQR model, we assumed no animal to human transmission in Bangladesh. The assumption is based on the observation that COVID-19 imported from overseas. As of March 28, there are 48 observed COVID-19 cases in Bangladesh; where, six of them have died, according to the Institute of Epidemiology, Disease Control, and Research [6]. The IEDCR'B claimed, out of 48 positive cases, 
there are 32 positive cases observed who traveled to Bangladesh from abroad. A significant portion of these 32 people either traveled in Europe or China, then returning to home or residing in Europe for a long time. So, it is our primary concern to heed on in-outboard travel passengers in Bangladesh in this paper. Then we will take into account what could happen if all those people quarantined, but somehow they have connected with relatives and friends surrounding him/her. We investigate what could happen if someone finished their 14-day quarantine but still carried the COVID-19 virus that is asymptomatic in his/her body, and he/she has contacted with another person. Finally, we will analyze all these scenarios mathematically and statistically.

\section{Model estimation}

We use the modified SEIR model with quarantine (Q) posted to medRxiv on 17 February 2020 [24]. We assumed that the virus transmitted among the travelers who had either traveled recently abroad from Bangladesh or returning home from Europe or Middle-East and then transmitted to others with whom they had close contact with them in various places of Bangladesh. Without proper regulations to keep these early travelers quarantined, the whole country is now at risk of contracting the novel coronavirus. (Fig. 1).

Based on Fig. 1 our proposed social distancing SEIQR model is the following:

$$
\begin{aligned}
\frac{d S(t)}{d t} & =\Lambda-s_{d} \beta S(t)((I(t)+k Q(t)) \\
& +\rho Q(t)-m_{p} S(t) \\
\frac{d E(t)}{d t} & =s_{d} \beta S(t)((I(t)+k Q(t)) \\
& -\left(\alpha_{1}+\alpha_{2}\right) E(t)-m_{p} E(t) \\
\frac{d I(t)}{d t} & =\alpha_{1} E(t)-\left(\delta+\gamma+m_{i}\right) I(t)-m_{p} I(t) \\
\frac{d Q(t)}{d t} & =\delta I(t)+\alpha_{2} E(t)-\omega Q(t)-\rho Q(t)-m_{p} Q(t) \\
\frac{d R(t)}{d t} & =\omega Q(t)+\left(\gamma+m_{i}\right) I(t)-m_{p} R 1(t)
\end{aligned}
$$

Where,

$$
\left(N, m_{p}, \Lambda, s_{d}, \alpha_{1}, \alpha_{2}, \rho, \omega, \delta, \gamma, m_{i}, k\right) \geq 0
$$

Parameter estimation: We named our model called the social distancing model SEIQR model. The transmissibility of COVID-19 based on this model based on the following assumptions or facts:

I) Individuals were assumed to fall in one of the five compartments: susceptible people $(\mathrm{S})$, exposed people (E), infected people (I), quarantined people $(\mathrm{Q})$ (who brought virus to Bangladesh but were not detected at the airport), and removed people (R) that states both recovered individual at the rate of $\gamma$ and dead individual at the rate of $m_{i}$. These two parameters play different rules in our developed model, and both do not affect the remaining individuals in the equation 1 . The usual death rate of peoples are defined as 
$m_{p}=0.00588$ (the death rate of Bangladesh is 5.88 per 1000 people [27]). In this model, we set $\Lambda=m_{p} \times N$, where $N=163443435$ refer to the total number of people in Bangladesh including peoples came from abroad. We also assume there are $80 \%$ infected people who have recovered by taking small treatment at a local hospital, and has died $1 \%$ infected people caused by the COVID-19. Thus, in this study, we have set $\gamma=0.80$ and $m_{i}=0.01$ for all analysis.

II) We assume that on March 7, 2020, before the first flight from Italy to Bangladesh, no positive cases have reported in Bangladesh. Travelers coming to Bangladesh who tested negative for COVID-19 by the initial screening test, and sent to the quarantine center. At that time, they have advised staying isolated for at least 14 days after quarantine. On March 17, an Indian newspaper reported that "One of the two has been infected with the virus after coming in contact with someone who had returned from abroad, IEDCR Director Prof Meerjady Sabrina Flora said" [29]Thus, in our model, we assume, at the beginning of March 2020, there was a lack of consciousness about COVID-19 in Bangladesh. Thus we assume, $k=0.90$ where $k \in[0,1]$ is the probability of people who got infected by the travelers in a specific area, and $70 \%$ of the isolated travelers who returned home on March 7, 2020 did not follow governmental request to be home quarantine [28], and came into contact with others.

III) We assume $\alpha_{1}=\frac{1}{4.0}$ as a rate of the latent incubation period, where mean incubation days are 4.0- days. Moreover, $\alpha_{2}=\alpha_{1}$ is the latent incubation rate home quarantined people; that is, on an average of 4.0- days as well. We assumed these parameters to track by our simulated data to the real data (see the Figure 2).

IV) We assume $\rho=\frac{1}{14}$ is the rate of quarantined people become susceptive because after 14-days some people thought they do not have any symptom of COVID-19, but it shows up after 14-days. Between this time, they have contact with other people around them, cause they wanted to meet with friends, known persons and relatives.

V) Our primary focus on the parameter $\omega$ the number of \% people of Bangladesh followed regulatory guidance and recovered from the quarantined center or the isolation situation. We will compare our result with the consideration of several \% people did not follow regulatory guidance and not remained himself/herself in home quarantined for the lockdown free days.

VI) In this research, we introduced a novel idea by introducing a social distancing parameter $s_{d}$ in our SEIQR model. We assumed this parameter value depends on how many people are making social interaction during lockdown free days. For example, if the government of Bangladesh locked the country for 7-days, and keep 23-days lockdown free, then it is likely to assume $75 \%$ will interact socially of these lockdown free days across the country. However, maybe $25 \%$ people of Bangladesh will be careful about the COVID - 19, and they did not go out that much, or they went outside but maintained social distance (at least $4 \mathrm{ft}$ ). On the other hand, when 7-days in lockdown during 30-days period, then people bound to kept themselves staying at home. So, we assume $90 \%$ people became very careful and kept themselves at home quarantine during the lockdown days. So that,

$$
\begin{aligned}
\delta & =\frac{1}{30} \times\left[\frac{90}{100} \times 7+\frac{10}{100} \times 23\right] \\
s_{d} & =\frac{1}{30} \times\left[\frac{75}{100} \times 23\right]
\end{aligned}
$$




\section{Basic Reproduction Number, $R 0$}

IIn this study, we used the $R_{0}$ to assess the transmissibility of the COVID-19. Commonly, $R_{0}$ was defined as the expected number of secondary infections that result from introducing a single infected individual into an otherwise susceptible population [19], [20], [21]. If $R_{0}>1$, the outbreak will occur. If $R_{0}<1$, the outbreak will toward an end. In this study, $R_{0}$ was deduced from the equation 1 model by the next generation matrix approach [22].

Based on the equations of our model, we can get the disease free equilibrium point as: $\left(\frac{\Lambda}{m_{p}}, 0,0,0,0\right)$

$$
F=\left[\begin{array}{ccc}
0 & \frac{s_{d} \beta \Lambda}{m_{p}} & \frac{s_{d} \beta \Lambda k}{m_{p}} \\
0 & 0 & 0 \\
0 & 0 & 0
\end{array}\right]
$$

$$
V=\left[\begin{array}{ccc}
\alpha_{1}+\alpha_{2}+m_{p} & 0 & 0 \\
-\alpha_{1} & \delta+\gamma+m_{i}+m_{p} & 0 \\
-\alpha_{2} & -\delta & \rho+\omega+m_{p}
\end{array}\right]
$$

$$
V^{-1}=\left[\begin{array}{ccc}
\frac{1}{\left(\alpha_{1}+\alpha_{2}+m_{p}\right)} & 0 & 0 \\
A & \frac{1}{\left(\delta+\gamma+m_{i}+m_{p}\right)} & 0 \\
B & C & \frac{1}{\left(\rho+\omega+m_{p}\right)}
\end{array}\right]
$$

where,

$$
\begin{aligned}
& A=\frac{\alpha_{1}}{\left(\alpha_{1}+\alpha_{2}+m_{p}\right)\left(\delta+\gamma+m_{i}+m_{p}\right)} \\
& B=\frac{\alpha_{1} \delta+\left(\delta+\gamma+m_{i}+m_{p}\right) \alpha_{2}}{\left(\alpha_{1}+\alpha_{2}+m_{p}\right)\left(\delta+\gamma+m_{i}+m_{p}\right)\left(\rho+\omega+m_{p}\right)} \\
& C=\frac{\delta}{\left(\delta+\gamma+m_{i}+m_{p}\right)\left(\rho+\omega+m_{p}\right)}
\end{aligned}
$$

$$
F V^{-1}=\left[\begin{array}{ccc}
D & E & F \\
0 & 0 & 0 \\
0 & 0 & 0
\end{array}\right]
$$


where,

$$
\begin{aligned}
D & =\frac{s_{d} \beta \Lambda\left(\left(k \alpha_{2}+\alpha_{1}\right) m_{p}+(k \delta+\omega+\rho) \alpha_{1}+k \alpha_{2}\left(\delta+\gamma 1+m_{i}\right)\right)}{m_{p}\left(\alpha_{1}+\alpha_{2}+m_{p}\right)\left(\delta+\gamma+m_{i}+m_{p}\right)\left(\rho+\omega+m_{p}\right)} \\
E & =\frac{s_{d} \beta \Lambda\left(k \delta+\omega+\rho+m_{p}\right)}{m_{p}\left(\delta+\gamma+m_{i}+m_{p}\right)\left(\rho+\omega+m_{p}\right)} \\
F & =\frac{s_{d} \beta \Lambda\left(k \delta+\omega+\rho+m_{p}\right)}{m_{p}\left(\delta+\gamma+m_{i}+m_{p}\right)\left(\rho+\omega+m_{p}\right)}
\end{aligned}
$$

Using matrix 7 we can find the basic reproduction number $R_{0}$ which is the spectral radius $\left(\rho^{*}\right)$ of the next generation matrix $F V^{-1}$. Thus,

$$
\begin{aligned}
R_{0} & =\rho^{*}\left(F V^{-1}\right) \\
& =\frac{s_{d} \beta \Lambda\left(\left(k \alpha_{2}+\alpha_{1}\right) m_{p}+(k \delta+\omega+\rho) \alpha_{1}+k \alpha_{2}\left(\delta+\gamma 1+m_{i}\right)\right)}{m_{p}\left(\alpha_{1}+\alpha_{2}+m_{p}\right)\left(\delta+\gamma+m_{i}+m_{p}\right)\left(\rho+\omega+m_{p}\right)}
\end{aligned}
$$

\section{Results}

Based on the parameter estimation as described before, first, we have studied the original data for COVID-19 positive cases from March 7, 2020, to March 28, 2020. Between these 22 - days, the IEDCR'B has tested 1024 people and found 48 people have COVID-19 positive. So, we initially assume the transmissibility rate $\beta=\frac{\frac{48}{1024 N}}{22}$. As of March 28, 2020, Bangladesh did not lock down the country. However, the Bangladesh government has declared a general vacation from March 26, 2020, two days back from March 28, 2020 [28]. So, we have assumed $L_{D}=2$ and $L_{F D}=20$. We assume people did not become cautious yet. Hence, we assume, $n_{Q}=10, n_{Q}^{*}=90$, where, $n_{Q}$ and $n_{Q}^{*}$ are represents out of 100 Bangladeshi, the number of quarantined and non-quarantined people respectively. Also, since the country did not put any pressure to move around freely, we assume there are $95 \%$ of the population of Bangladesh are making social interaction within various places, i.e., $n_{d}=95$. With these assumptions, we fit our simulated result with the real data [6]. A linear regression polynomial fit results with $\left(R^{2}=0.9320\right)$, with adjusted $\left(R^{2}=0.9250\right)$ (Fig. 2) showed that our model fitted well to the reported COVID-19 cases data for Bangladesh. Now, using all the assumptions and the equation 9 , we have estimated the value of the basic reproduction number $R_{0}=4.16$, which means, we may say, within these 22-days COVID19 coronavirus is already an outbreak in Bangladesh. For all parameter details, please see the table 1.

\section{Discussions}

This study aimed to find an appropriate mathematical model, and compare the results with the real cases. The second approach is to find some strategies that can help Bangladesh to reduce the transmission of the coronavirus. A mathematical model is useful to find a basic reproduction number, $R_{0}$ for a disease. Our main goal is to decrease the value of $R_{0}$ 
using our mathematical model to find those strategies by considering 'lockdown,' 'social distancing' and 'quarantine.'

Figure 3 is showing that there is a high chance for the COVID-19 coronavirus can spread in the high number of people in Bangladesh around in mid-April, 2020. So far, people already became cautious. However, still, the country has much other concern to face this COVID19 coronavirus at many levels. Suppose there is no lockdown at any place in Bangladesh that is assumed, $L_{D}=0$, and $L_{F D}=30$ now. Since people have a chance to go out and make social interaction freely, we assume $90 \%$ peoples of Bangladesh will make social interaction with each other, where $10 \%$ people are self cautious and kept themself at-home quarantine. Also suppose, $\omega=10 \%$, the inboard passenger sent to the quarantine center, and presumably, they all removed from the quarantine center. From this situation, when a country has limited medical resources to test the vast amount of people of the country's populations and provide intensive medical treatment, any third-world country can follow three well guidance provided by WHO, which are:

Case-I) Take administrative action (i.e., Applying lockdown the country)

Case-II) Bangladeshi can take responsibility by themselves (i.e., ensuring to maintain social distance rule by the WHO guidance )

Case-III) Ensuring the nation to stay at home (i.e., ensuring people to keep themselves either at home quarantine or governmental quarantine center)

We will show some mathematical and statistical results that can be a direction for a thirdworld country to reduce the infectious number of people.

First, we will discuss what administrative authority of a lower economic-based country may consider doing for preventing COVID-19 coronavirus from spreading across the country. A long days lockdown can cost huge for any country, so assume a developing cannot effort economically by making lockdown the country for more than a month. However, to make the situation under control, they can try to make lockdown the country from one week to three weeks. So, we now assume $n_{Q}=10+2.5 \times L_{D}$, and $n_{d}=95-2.5 \times L_{F D}$. The basic reproduction number $R_{0}=5.72$ when the entire country is not lockdown yet. So that, they can think to consider making lockdown the country for one week. Figure 4 and Table 2 shows that, seven days lockdown is not sufficient to control spreading the virus. Nevertheless, as we found, it may need at least three weeks lockdown to reduce the reproduction of this COVID-19 in Bangladesh and protect the nation from affecting.

Also, a Pearson's correlation was run to determine the relationship between five cases (zero days, one, two, three, and complete thirty days lockdown situation) between each other. There was a robust, positive correlation on the infective numbers between one, two, three weeks lockdown situation, and complete lockdown situation. (see: figure 4).

The Pearson's correlation result shows that when there is a limited resource to control an outbreak, then at least three weeks lockdown the country could be an excellent strategy to reduce the number of infections.

But, for a developing country like Bangladesh, three weeks lockdown could put the country in the economic hardship. Especially when a country has 5-millions of people who earned less than $\$ 2$ per-day. From this viewpoint, we now focused on decreasing the rate of social distancing parameter $s_{d}$. We have run our model by setting this parameter values from 
0.50 to 0.10 with an interval of 0.10 . That means we are thinking during the lockdown free situation, Bangladeshi people will make social gathering on an average of 50\% people at the beginning. Table- 3 shows that when an average of $50 \%$ people are making social interaction can create a massive outbreak situation for the country. On the other hand, when several percentiles of people in Bangladesh, when making social interaction on an average of $\leq 10 \%$ can reduce the outbreak of this COVID - 19 in Bangladesh.

Several authors suggest, when an epidemical disease become pandemic and especially when there is no vaccination is useful, then it is highly suggestive that sent people to the home quarantine. Researchers suggests that, using this procedure a country can increase the recovery rate [20],[21],[22]. We assume Bangladesh can also follow this procedure to protect the nation from this virus. Suppose now, there is a 50\% probability that home quarantined peoples have become susceptive, and 35\% population of Bangladesh quarantined, and $75 \%$ people were making social interactions at the beginning. That is we assumes $n_{Q}=35, n_{d}=75$ and $k=0.5$. From this point, we have used our model to find $R_{0}$ by varying the parameter $\omega$. We have started from $10 \%$ to $60 \%$ by considering the entire country is not locked officially, but the people took responsibility and made themself isolated or kept themself as a home quarantined person. Our modeling simulation shows that it changes the result impressively. Table- 4 shows that at this time, if $60 \%$ or higher people of Bangladesh kept themselves at home at least for 30 days, then $R_{0}<1$, which indicates the outbreak will toward an end in Bangladesh.

Apart from some essential parameters $\left(\omega, s_{d}, \delta\right)$, from the equation 7 , we can see, there are other parameters also plays an important rule to decrease the $R_{0}$ value. Such as, increasing incubation period may decrease the infective people, which can decrease the basic reproduction number as well.

\section{Restrictions}

Since based on our model, $R_{0}$ depends on one many parameters. That needs more real data to predict more accurately. When we are researching for the case of Bangladesh, we have not found some critical parameter values, such as, we do not know what the probability ( $k$ - in our model) that quarantined people become susceptive is. So, we assumed it is 50\%. However, it can be $0 \%$ or can be $100 \%$. At this time, we do not know what the mean period that we can say correctly in how many days or hours quarantined people is becoming susceptive is. So, we have assumed it is on an average of seven days $\left(\rho=\frac{1}{14}\right)$. However, the real situation can be much worse or much better.

\section{Conclusions}

COVID-19 coronavirus shows that currently, there is a serious concern of the outbreaking situation in Bangladesh to spread this novel coronavirus in a huge number of population. Bangladesh has limited resources to face this outbreak. At this time, a robust strategy can control faster from spreading the virus and protect the nation. We have found, a three weeks lockdown can decrease the reproduction number of this novel coronavirus from now on (see figure-5(top)). We also have shown, for a developing country like Bangladesh, mass peoples are also responsible for reducing this virus by following the WHO guidance and following social distance (see figure-5(middle)). Also, we have found people can take the 
responsibility to make their home as a quarantine place that could decrease spreading the virus rapidly (see figure-5 (bottom)).

\author{
Competing interests \\ The authors declare that they have no competing interests.
}

Author's contributions

All authors contributed equally to complete this research.

\title{
Acknowledgements
}

Not Applicable.

Funding

This study was partially supported by Grant-in-Aid for Scientific Research from JSPS, Japan, KAKENHI (Grant No. JP 19KK0262) awarded to Professor Tanimoto.

Ethical Approval and Consent to participate

Not Applicable.

Consent to for publication

Not Applicable.

Availability of Supporting data

https://figshare.com/articles/Data_and_code_on_the_effects_of_quarantine_and_social_distancing_on_the_ outbreak_of_novel_coronavirus_in_developing_or_under_poverty_level_countries_/12123681

\section{Author details}

${ }^{1}$ College of Business, Economics, Applied Statistics and International Business, New Mexico State University, MSC 3CQ, PO Box 30001 Las Cruces, NM 88003-8001, U.S.A. ${ }^{2}$ Interdisciplinary Graduate School of Engineering Sciences, Kyushu University, Kasuga-koen, Kasuga-shi, 816- 8580 Fukuoka, Japan. ${ }^{3}$ Department of Mathematics, Bangladesh University of Engineering and Technology, Dhaka, Bangladesh. ${ }^{4}$ Faculty of Engineering Sciences, Kyushu University, Kasuga-koen, Kasuga-shi, 816- 8580 Fukuoka, Japan.

\section{References}

1. World Health Organization. Coronavirus. World Health Organization cited January 19, 2020. Available: https://www.who.int/health-topics/coronavirus.

2. Zhou $P$, Yang XL, Wang XG, Hu B, Zhang L, Zhang W, et al. A pneumonia outbreak associated with a new coronavirus of probable bat origin. Nature. 2020. https://doi.org/10.1038/s41586-020-2012-7.

3. Huang C, Wang Y, Li X, Ren L, Zhao J, Hu Y, et al. Clinical features of patients infected with 2019 novel coronavirus in Wuhan, China. Lancet. 2020. https://doi.org/10.1016/S0140-6736(20)30183-5.

4. Li Q, Guan X, Wu P, Wang X, Zhou L, Tong Y, et al. Early transmission dynamics in Wuhan, China, of novel coronavirus-infected pneumonia. N Engl J Med. 2020.

5. https://en.wikipedia.org/wiki/ 2020 coronavirus pandemic in Bangladesh.

6. https://www.iedcr.gov.bd

7. Kermack, W.O., McKendrick, A.G.; A contribution to the mathematical theory of epidemics. Proc. Roy. Soc. Of London, Series A 115,1927,700-721.

8. May, Robert M.; Anderson, Roy M. Infectious diseases of humans: dynamics and control. Oxford: Oxford University Press, 1991. ISBN 0-19-854040-X.

9. Li, M. Y., Muldowney, J. S.; Global stability for the SEIR model in epidemiology, Math. Bio.,125 (2), 1995,155-164.

10. Feng, Z. Final and Peak Epidemic Sizes for SEIR Models with Quarantine and Isolation. Math. Biosci. Eng. 2007, 4, 675-686.

11. Safi, M.A.; Imran, M.; Gumel, A.B. Threshold dynamics of a non-autonomous SEIRS model with quarantine and isolation. Theory Biosci. 2012, 131, 19-30.

12. Yongzhen, P., Shaoying, L., Shuping, L., Changguo, L.; A delayed SEIQR epidemic model with pulse vaccination and the quarantine measure, Comp. and Math. with App., 2009, 58:135-145

13. Kabir, KM. A., Kuga, K., Tanimoto, J.; Analysis of SIR epidemic model with information spreading of awareness, Cha. Sol. and Frac. 2019, 119, 118-125

14. Kabir, KM. A., Tanimoto, J.; Analysis of epidemic outbreaks in two-layer networks with different structures for information spreading and disease diffusion, Comm. Non. Sci. Nun. Sim. 2009, 72, 565-574.

15. Kabir, K. M. A., Tanimoto, J.; Impact of awareness in metapopulation epidemic model to suppress the infected individuals for different graphs, European Physical Journal B 2019, 92, 199.

16. Kabir, K. M. A., Jusup, M., Tanimoto, J.; Behavioral incentives in a vaccination-dilemma setting with optional treatment, Physical Review E 2019, 100, 062402.

17. Alam, M., Kabir, K. M. A., Tanimoto, J.; Based on mathematical epidemiology and evolutionary game theory, which is more effective: quarantine or isolation policy? Jstate, 2020, 033502, https://doi.org/10.1088/1742-5468/ab75ea.

18. G. A. Ngwa and M. I. Teboh-Ewungkem, A mathematical model with quarantine states for the dynamics of Ebola virus disease in human populations, Comput. Math. Methods Med. 2016, 9352725 
19. Chen T, Ka-Kit Leung R, Liu R, Chen F, Zhang X, Zhao J, et al. Risk of imported Ebola virus disease in China. Travel Med Infect Dis. 2014;12:650-8.

20. Zhang S, Hu Q, Deng Z, Hu S, Liu F, Yu S, et al. Transmissibility of acute haemorrhagic conjunctivitis in small-scale outbreaks in Hunan Province, China. Sci Rep. 2020;10:119

21. Chen S, Yang D, Liu R, Zhao J, Yang K, Chen T. Estimating the transmissibility of hand, foot, and mouth disease by a dynamic model. Public Health. 2019;174:42-8.

22. Cui J-A, Zhao S, Guo S, Bai Y, Wang X, Chen T. Global dynamics of an epidemiological model with acute and chronic HCV infections. Appl Math Lett. 2020;103:106203.

23. Kissler, Stephen M. and Tedijanto, Christine and Goldstein, Edward and Grad, Yonatan H. and Lipsitch, Marc, Projecting the transmission dynamics of SARS-CoV-2 through the postpandemic period, Science, 2020 https://science.sciencemag.org/content/early/2020/04/24/science.abb5793.

24. Liangrong Peng, Wuyue Yang, Dongyan Zhang, Changjing Zhuge, and Liu Hong, Epidemic analysis of COVID-19 in China by dynamical modeling, medRxiv, 2020. https://doi.org/10.1101/2020.02.16.20023465

25. Chen, T., Rui, J., Wang, Q. et al. A mathematical model for simulating the phase-based transmissibility of a novel coronavirus. Infect Dis Poverty 2020, 9, 24 https://doi.org/10.1186/s40249-020- 00640-3

26. Li Q, Guan X, Wu P, Wang X, Zhou L, Tong $Y$, et al. Early transmission dynamics in Wuhan, China, of novel coronavirus-infected pneumonia. N Engl J Med. 2020. https://doi.org/10.1056/NEJMoa2001316

27. https://knoema.com/atlas/Bangladesh/Death-rate

28. https://www.atlanticcouncil.org/blogs/new-atlanticist/defusing-bangladeshs-covid-19-timebomb/

29. https://www.indiatoday.in/world/story/bangladesh-coronavirus-cases-rise-to-10-govt-urges-people-to-followquarantine-guidelines-1656574-2020-03-17 


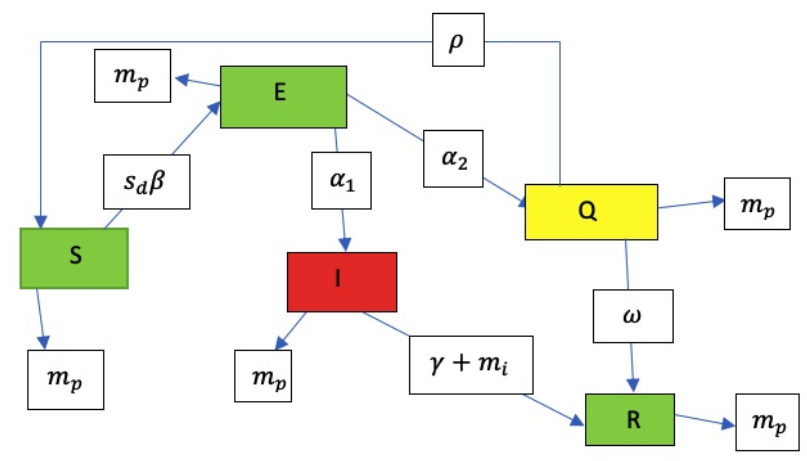

Figure 1 Flowchart of the modified SEIR dynamics for COVID-19 coronavirus in developing countries including quarantine $(Q)$ and social distancing situation

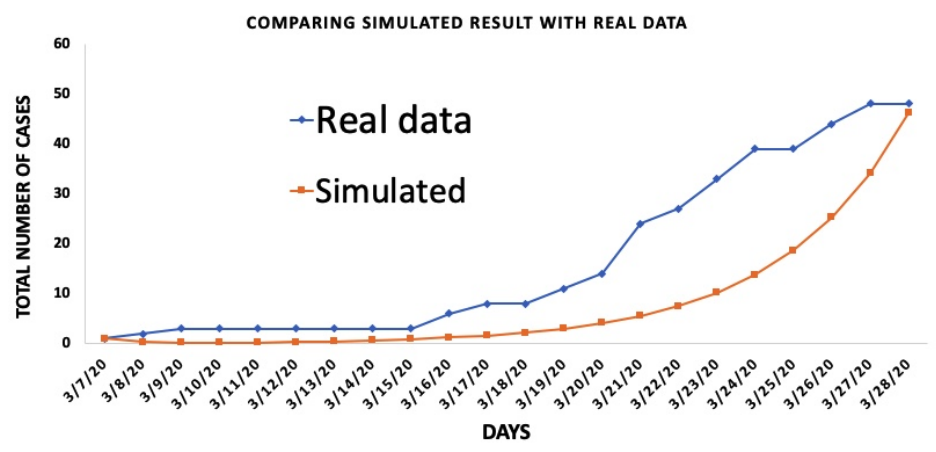

Figure 2 Simulated result compared with real data [5] March 7,2020 to March 28, 2020. A linear regression polynomial fit curve used for the simulated data with $R^{2}=0.9320$

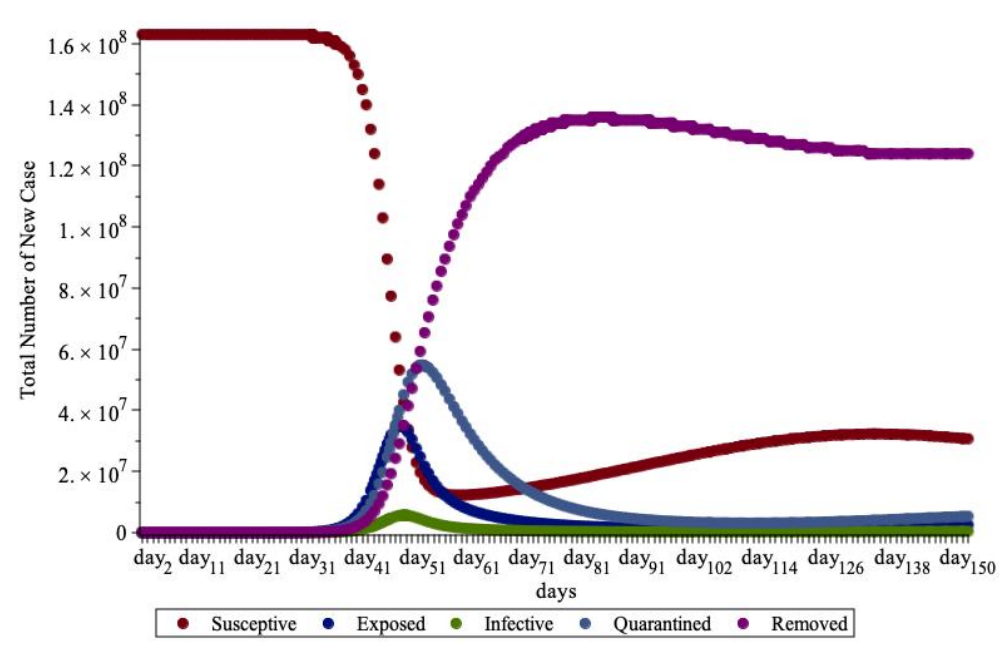

Figure 3 Based on our model we are predicting there is a chance that COVID-19 coronavirus will be it's pick time to spread throughout the country after 40- days from March 7,2020 in Bangladesh. 

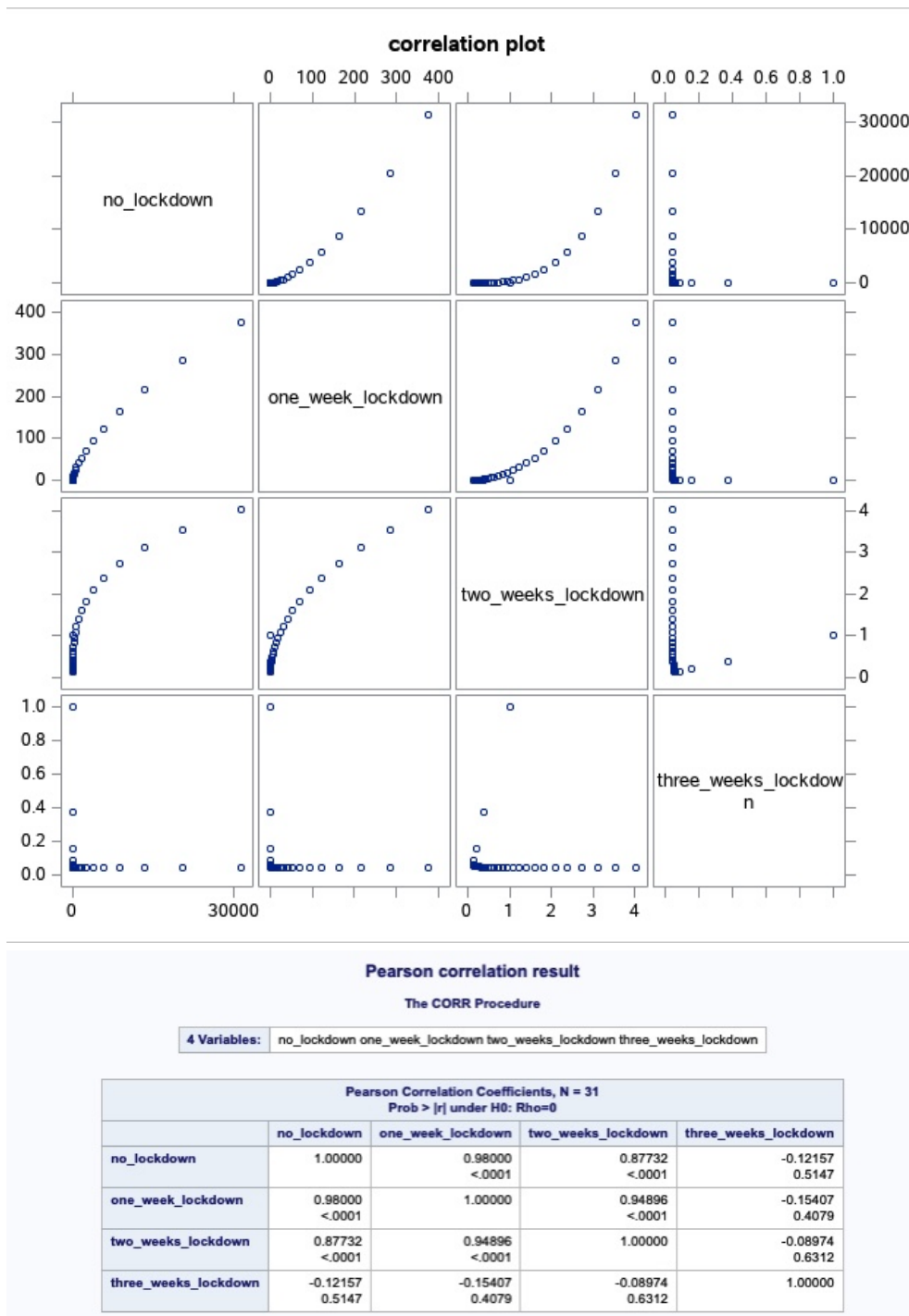

Figure 4 Pearson's correlation results presented here in a lockdown situation in Bangladesh. Results show that at zero-days, lockdown (Lockdown-0) versus one-week lockdown (lockdown-7) situation has a powerful positive correlation with $p-v a l u e<0.0001$. That means if the infected number of people increases for one week lockdown, in a one-week lockdown situation number of infected people will increase too. But, we can also see there is a negative correlation between zero days lockdown situation and three weeks lockdown situation with high $p-v a l u e$, that means statistically there no significant evidence that if the number of infected people increases during the zero-days lockdown time, a three weeks lockdown will also have chance to increase the number of infected persons 

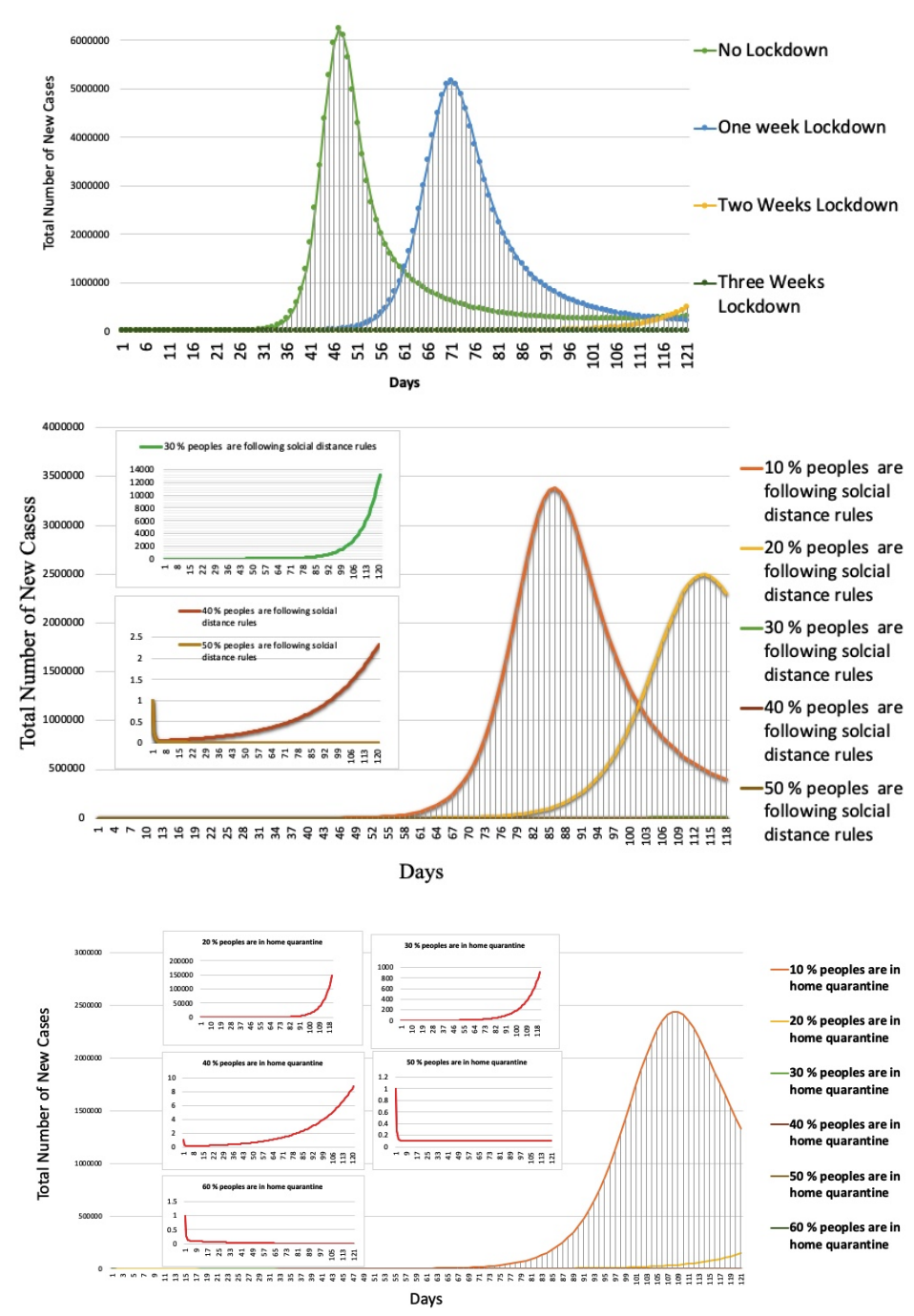

Figure 5 (top) Based on our model showing the result for Case-I. (middle) showing the result for Case-II, and (bottom) showing the result for Case-III.

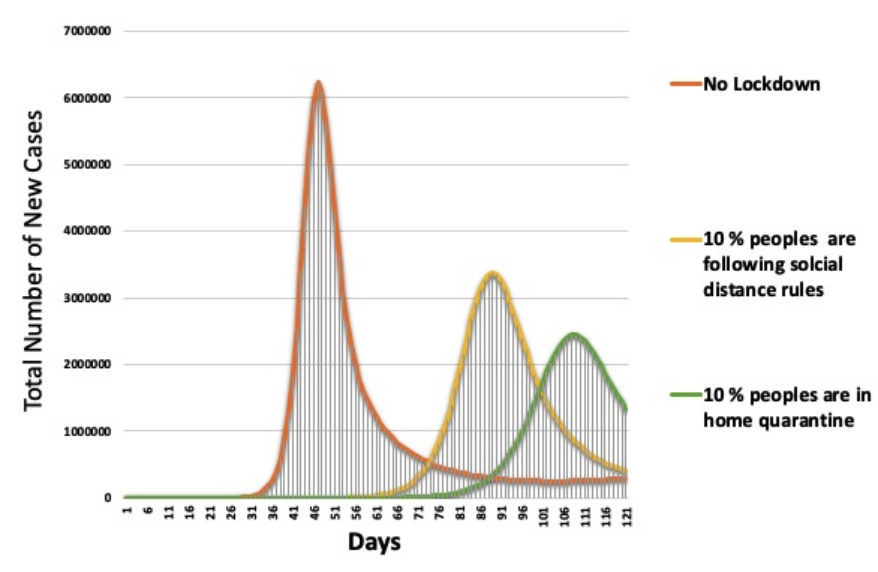

Figure 6 Comparison between all three cases, this figures indicates that among those three cases staying home can provide better result to protect people from infections from the coronavirus. 
Table 1 Definition of those parameters in the SEIQR social distancing model:

\begin{tabular}{lll}
\hline Parameter & Description & Values \\
\hline$N$ & Total population of Bangladesh & 163443435 \\
$m_{p}$ & Death rate & 0.00588 \\
$m_{i}$ & Mortality rate of the infected people & $\frac{1}{100}$ \\
$\gamma$ & Rate of infected people become recovered & $\frac{80}{100}$ \\
$\Lambda$ & Birth rate of Bangladesh & $m_{p} \times N$ \\
$\beta$ & Transmissibility rate & Vary \\
$\alpha_{1}$ & Latent incubation rate to be infectctive & $\frac{1}{7}$ \\
$\alpha_{2}$ & Latent incubation rate to be quarantined & $\alpha_{1}$ \\
$\rho$ & Rate of recovered people become susceptive & $\frac{1}{14}$ \\
$\omega$ & Rate of quarantined people become recovered or dead & $\operatorname{Vary}$ \\
$\delta$ & Rate of infected people become quarantined & $\frac{1}{L_{D}+L_{F D}} \times\left[\frac{n_{Q}}{100} \times L_{D}+\frac{n_{Q}^{*}}{100} \times L_{F D}\right]$ \\
$n_{Q}$ & Number of quarantined people out of 100 & Vary \\
$n_{Q}^{*}$ & Number of non-quarantined people out of 100 & $n_{Q}^{*}=100-n_{Q}$ \\
$L_{D}$ & Number of Lockdown days in Bangladesh & Vary \\
$L_{F D}$ & Number of Lockdown free days in Bangladesh & Vary \\
$n_{d}$ & Number of people making social interaction out of 100 & Vary \\
$s_{d}$ & Social distancing parameter & $\frac{1}{L_{D}+L_{F D}} \times\left[\frac{n_{d}}{100} \times L_{F D}\right]$ \\
$k$ & The multiple of the transmissibility of $Q(t)$ to that of $I(t)$ & {$[0,1]$} \\
\hline
\end{tabular}

Table 2 Prediction regarding lockdown situation:

\begin{tabular}{|l|c|c|c|c|}
\hline Description & parameter & values & $R_{0}$ & Prediction \\
\hline No & $\delta$ & 0.9 & & \\
lockdown & $s_{d}$ & 0.95 & 5.93 & outbreak \\
& $\beta$ & $8.67 \times 10^{-09}$ & & \\
\hline one week & $\delta$ & 0.6387 & & \\
lockdown & $s_{d}$ & 0.621 & 3.77 & outbreak \\
& $\beta$ & $8.69 \times 10^{-09}$ & & \\
\hline two & $\delta$ & 0.508 & & \\
weeks & $s_{d}$ & 0.357 & 2.12 & Outbreak \\
lockdown & $\beta$ & $8.66 \times 10^{-09}$ & & \\
\hline Three & $\delta$ & 0.508 & & \\
weeks & $s_{d}$ & 0.16 & 0.945 & Stable \\
lockdown & $\beta$ & $8.71 \times 10^{-09}$ & & \\
\hline
\end{tabular}

Table 3 Prediction regarding maintaining social distancing by isolating the people across the country:

\begin{tabular}{|c|c|c|c|c|}
\hline Description & parameter & values & $R_{0}$ & Prediction \\
\hline \multirow{4}{*}{ Zero days lockdown } & $s_{d}$ & 0.50 & 3.13 & outbreak \\
& $s_{d}$ & 0.40 & 2.50 & outbreak \\
& $s_{d}$ & 0.30 & 1.87 & outbreak \\
& $s_{d}$ & 0.20 & 1.27 & outbreak \\
& $s_{d}$ & 0.10 & 0.623 & Stable \\
\hline
\end{tabular}

Table 4 Prediction regarding quarantined people become recovered person:

\begin{tabular}{|l|c|c|c|}
\hline Description & recovery rate of quarantined people & $R_{0}$ & Prediction \\
\hline \multirow{2}{*}{ Zero } & $10 \%$ people & 2.47 & outbreak \\
days & $20 \%$ people & 1.70 & outbreak \\
lockdown & $30 \%$ people & 1.35 & outbreak \\
& $40 \%$ people & 1.14 & outbreak \\
& $50 \%$ people & 1.00 & outbreak \\
& $60 \%$ people & 0.905 & stable \\
\hline
\end{tabular}




\section{Figures}

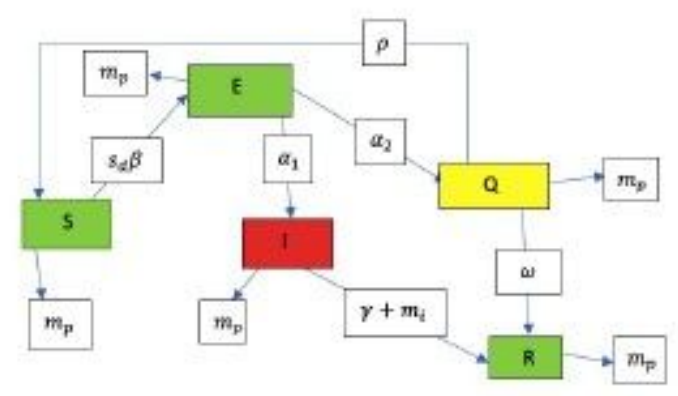

Figure 1 Flowchart of the modified SEIR dynamic for COVID-19 coronavirus in developing countries induding quarantine $(Q)$ and social distancing situation

\section{Figure 1}

Flowchart of the modified SEIR dynamics for COVID-19 coronavirus in developing countries including quarantine $(\mathrm{Q})$ and social distancing situation

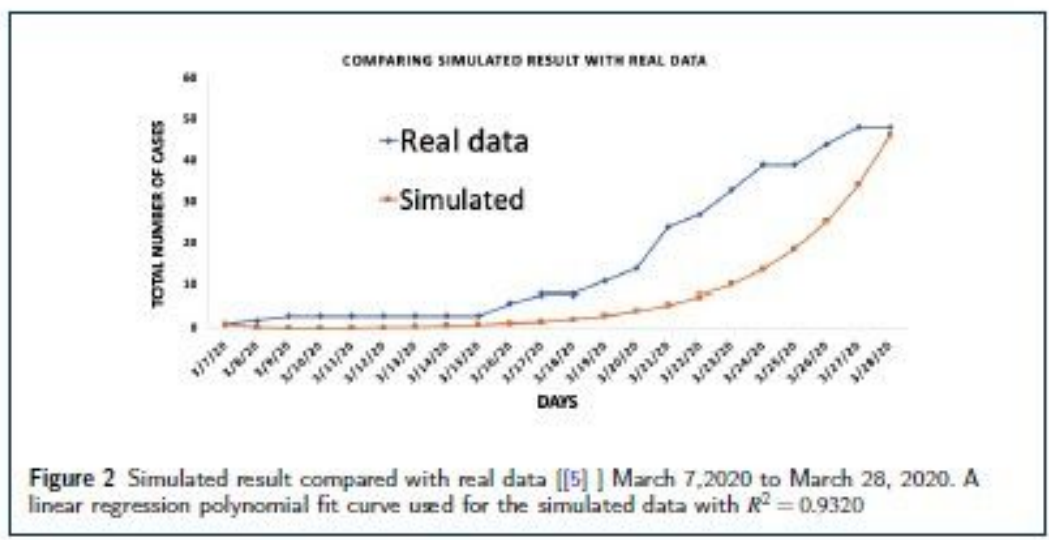

\section{Figure 2}

Simulated result compared with real data [5] March 7,2020 to March 28, 2020. A linear regression polynomial fit curve used for the simulated data with $\mathrm{R} 2=0.9320$ 


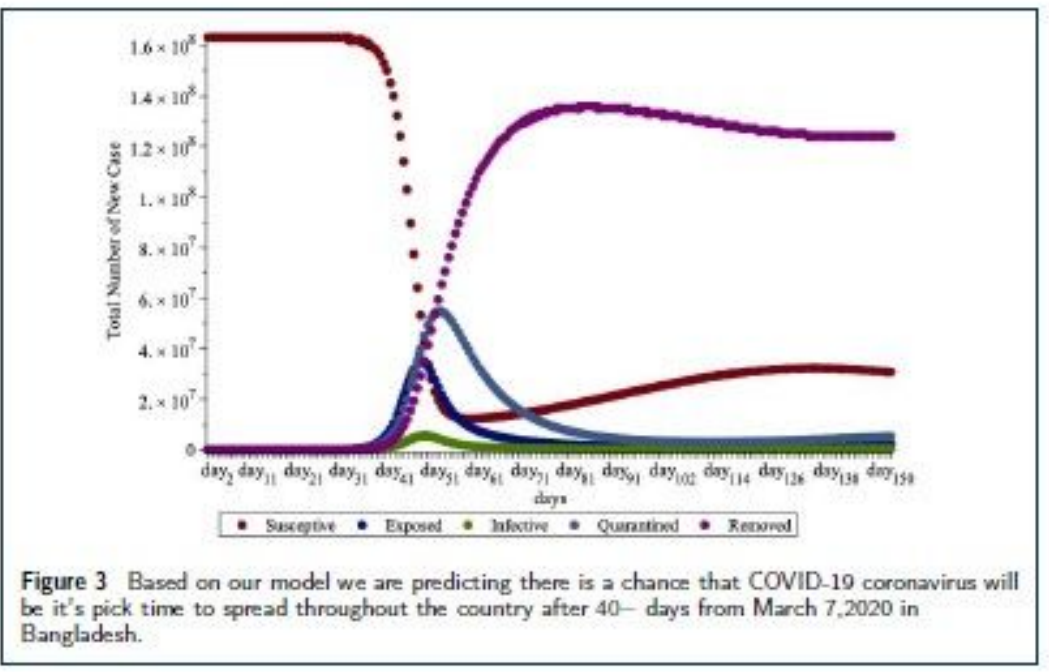

Figure 3

Based on our model we are predicting there is a chance that COVID-19 coronavirus will be it's pick time to spread throughout the country after 40- days from March 7,2020 in Bangladesh. 


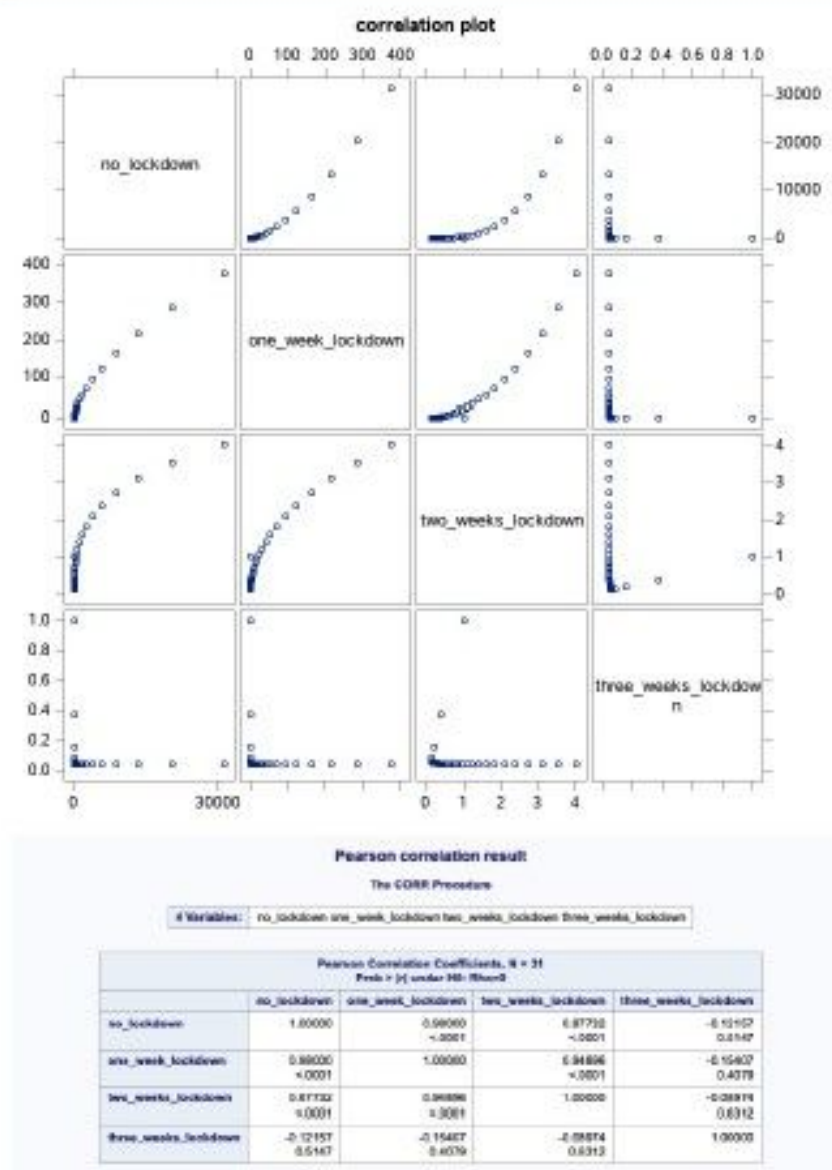

Figure 4 The Pearson's correlation result for lockdown situation in Bangladesh. Results shows that, at zero days lockdown (Lookdown-0) versus one week lockdown (lockdown-7) situation has very strong positive correlation with $p-v a l u e<0.0001$. That means, if the infected number of people increases for one week lockdown, in one week lockdown situation number of infected people will increase too. But, we can also see there is a negative correlation between zero days lockdown situation and three weeks lockdown situation with high $p$-valwe, that means statistically there no significance evidence that if the number of infected people increases during the zero days lockdown time, a three weeks lockdown will also have chance to increase the number of infected persons

\section{Figure 4}

Pearson's correlation results presented here in a lockdown situation in Bangladesh. Results show that at zero-days, lockdown (Lockdown-0) versus one-week lockdown (lockdown-7) situation has a powerful positive correlation with $p$-value $<0.0001$. That means if the infected number of people increases for one week lockdown, in a one-week lockdown situation number of infected people will increase too. But, we can also see there is a negative correlation between zero days lockdown situation and three weeks lockdown situation with high p-value, that means statistically there no significant evidence that if the number of infected people increases during the zero-days lockdown time, a three weeks lockdown will also have chance to increase the number of infected persons 


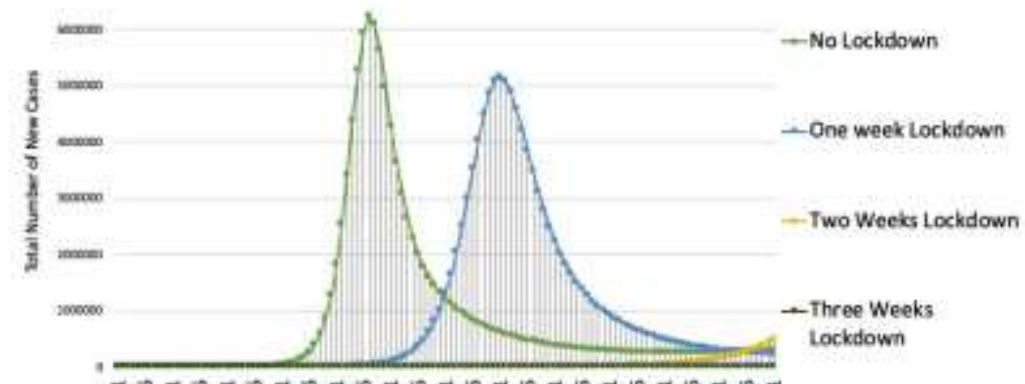

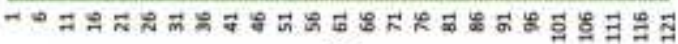

Dans
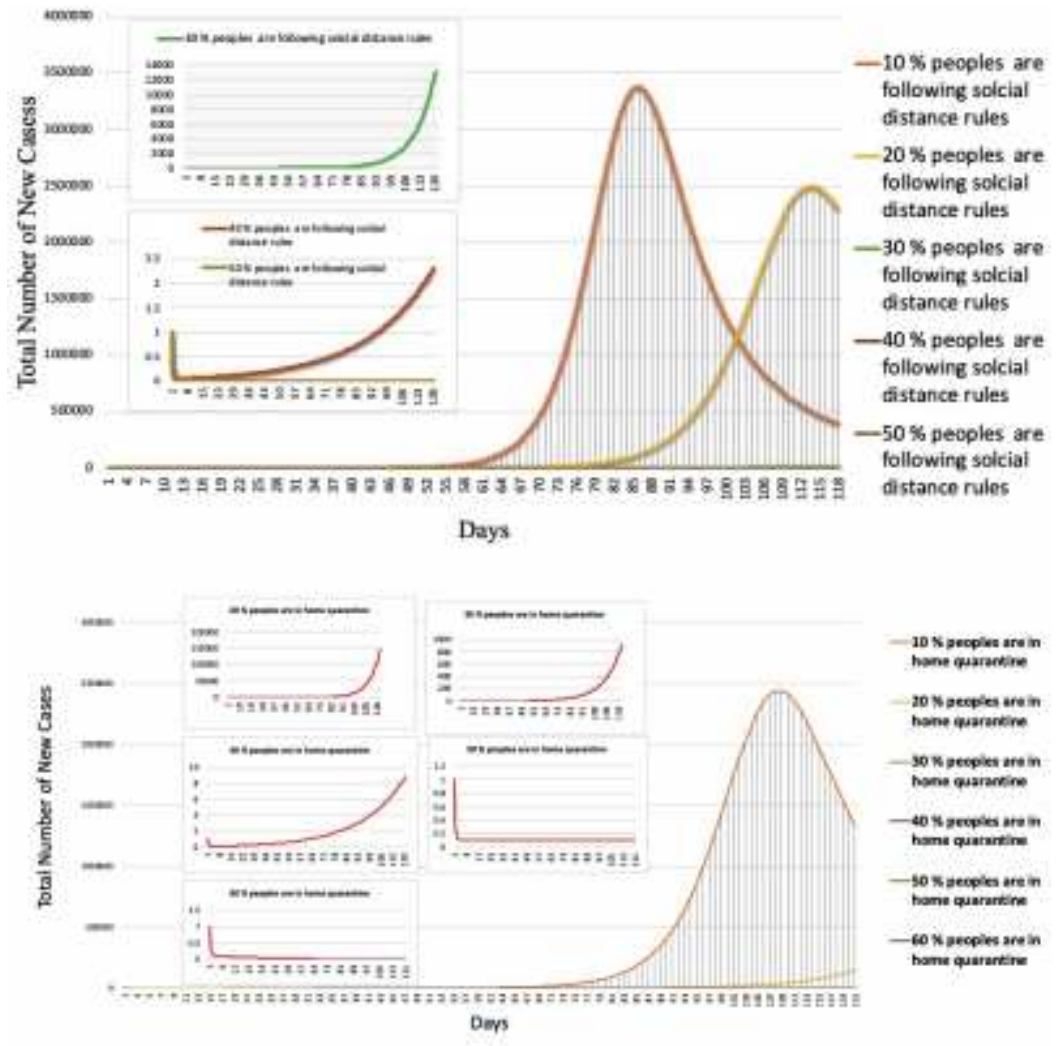

Figure 5

(top) Based on our model showing the result for Case-I. (middle) showing the result for Case-Il, and (bottom) showing the result for Case-Ill. 


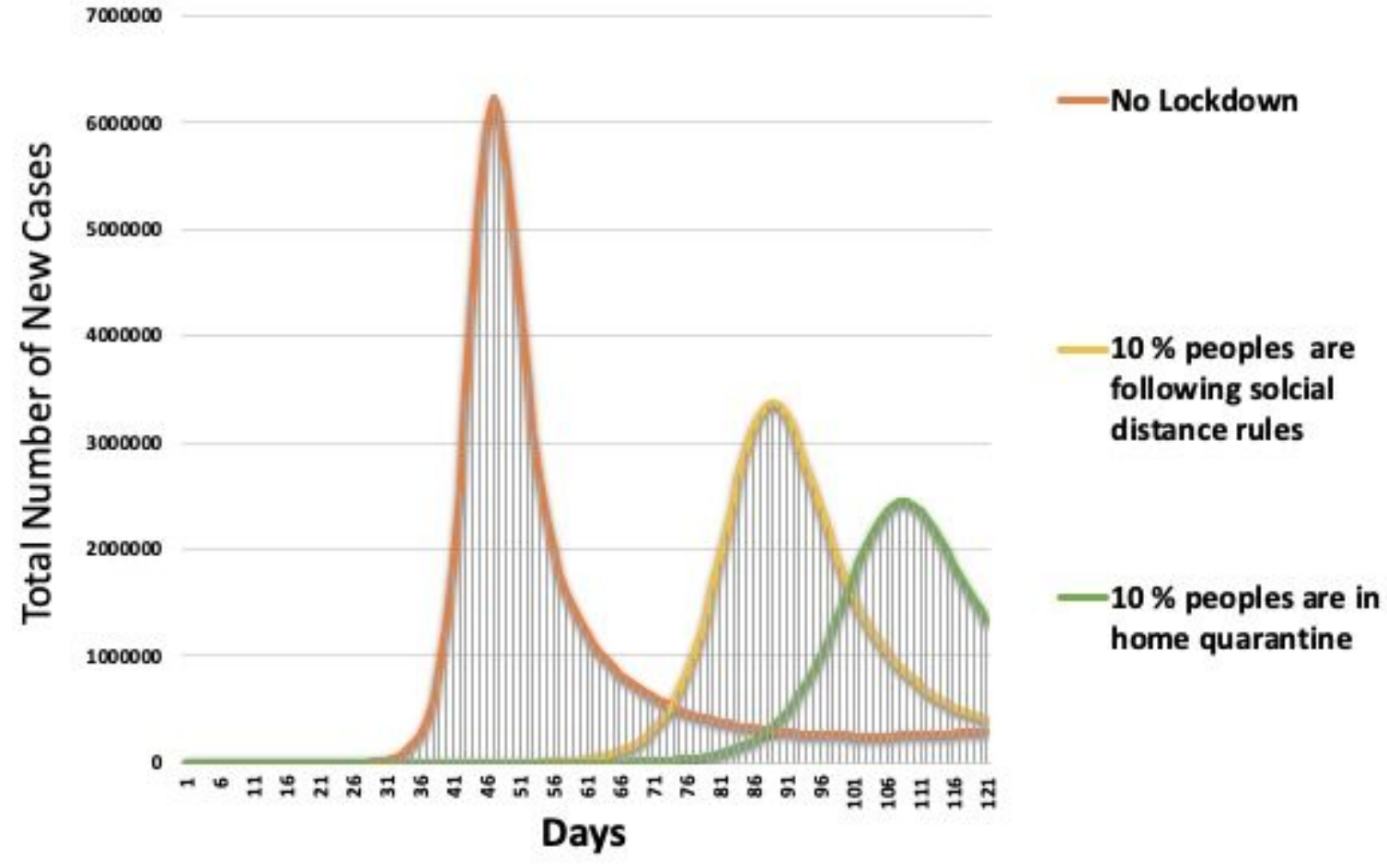

Figure 6

Comparison between all three cases, this figures indicates that among those three cases staying home can provide better result to protect people from infections from the coronavirus. 